\title{
Sensory and Motor Behavior Evidences Supporting the Usefulness of Conditioned Medium from Dental Pulp-Derived Stem Cells in Spinal Cord Injury in Rats
}

\author{
Reza Asadi-Golshan ${ }^{1,2,3}$, Vahid Razban ${ }^{4}$, Esmaeil Mirzaei ${ }^{5}$, Abdolkarim Rahmanian ${ }^{6}$, \\ Sahar Khajeh ${ }^{7}$, Zohreh Mostafavi-Pour ${ }^{8}$, Farzaneh Dehghani ${ }^{1,2}$ \\ ${ }^{I}$ Department of Anatomy, School of Medicine, Shiraz University of Medical Sciences, Shiraz, Iran \\ ${ }^{2}$ Histomorphometry and Stereology Research Centre, Shiraz University of Medical Sciences, Shiraz, Iran \\ ${ }^{3}$ Cellular and Molecular Medicine Student Research Group, School of Medicine, Shiraz University of Medical Science, Shiraz, Iran \\ ${ }^{4}$ Department of Molecular Medicine, School of Advanced Medical Sciences and Technologies, Shiraz University of Medical Sciences, Shiraz, Iran \\ ${ }^{5}$ Department of Medical Nanotechnology, School of Advanced Medical Sciences and Technologies, Shiraz University of Medical Sciences, Shiraz, Iran \\ ${ }^{6}$ Department of Neurosurgery, Shiraz University of Medical Sciences, Shiraz, Iran \\ ${ }^{7}$ Department of Biochemistry, School of Medicine, Shiraz University of Medical Sciences, Shiraz, Iran \\ ${ }^{8}$ Recombinant Protein Laboratory, Department of Biochemistry, School of Medicine, Shiraz University of Medical Sciences, Shiraz, Iran
}

Study Design: Experimental animal study.

Purpose: This study aimed to assess effects of conditioned medium (CM) of dental pulp-derived stem cells loaded in collagen hydrogel on functional recovery following spinal cord injury (SCI).

Overview of Literature: $\mathrm{SCl}$ affects sensory and motor functions, and behavioral recovery is the most essential purpose of therapeutic intervention. Recent studies have reported that CM from dental pulp-derived stem cells has therapeutic benefits. In addition, collagen hydrogel acts as a drug delivery system in SCl experiments.

Methods: Stem cells from human exfoliated deciduous teeth (SHEDs) were cultured, and SHED-CM was harvested and concentrated. Collagen hydrogel containing SHED-CM was prepared. The rats were divided into five groups receiving laminectomy, compressive SCI with or without intraspinal injection of biomaterials (SHED-CM), and collagen hydrogel with or without SHED-CM. Basso, Beattie, and Bresnahan (BBB) scoring, inclined plane, cold allodynia, and beam walk tests were performed for 6 weeks to assess locomotor, motor, sensory, and sensory-motor performances, respectively.

Results: Scores of the rats receiving SHED-CM loaded in collagen hydrogel were significantly better than those of the other injured groups at 1-week post-injury for BBB, 2 weeks for inclined plane, 2 weeks for cold allodynia, and 4 weeks for beam walk tests $(p<0.05)$. The differences remained significant throughout the study.

Conclusions: Intraspinal administration of SHED-CM loaded in collagen hydrogel leads to improved functional recovery and proposes a cell-free therapeutic approach for SCl.

Keywords: Spinal cord injuries; Collagen; Conditioned culture media; Behavior; Rats

Received Nov 14, 2017; Revised Mar 27, 2018; Accepted Apr 17, 2018

Corresponding author: Farzaneh Dehghani

Department of Anatomy, School of Medicine, Shiraz University of Medical Sciences, Zand Blvd, Shiraz, Iran

Tel: +98-71-32304372, Fax: +98-71-32304372, E-mail: dehghanf@sums.ac.ir 


\section{Introduction}

Spinal cord injury (SCI) affects motor, sensory, and autonomic functions due to changes at the injury site. These changes lead to limited tissue regeneration [1]. Behavioral recovery following SCI is one of the most important purposes of therapeutic intervention and is related to the severity of damage [2]. Functional recovery is the key factor in clinical investigations regarding a therapeutic approach for SCI [3].

Dental pulp-derived stem cells have multiple potential uses; they are simple to extract and collect and show rapid proliferation. Differentiation of these stem cells into neural-like cells has been demonstrated in vitro $[4,5]$. Recently, transplantation of stem cells from human exfoliated deciduous teeth (SHEDs) after experimental SCI has shown improved motor functional recovery [6,7]. However, there are some disadvantages related to cell therapy, such as tumorigenesis and immune reactions [8]. A useful approach to overcome the difficulties in cell transplantation is the use of cell-derived conditioned medium (CM). Recent studies have reported that CM derived from dental pulp stem cells improves bone healing [9], promotes cognitive function in Alzheimer's disease [10], and reduces cardiac injury after ischemia reperfusion [8]. In this study, we speculated that SHED-CM could be effective in functional recovery following SCI.

Collagen hydrogel has many benefits, such as good biocompatibility and appropriate biodegradability. The effectiveness of the collagen scaffold as a drug delivery system has been shown in several animal SCI experiments [1113]. For example, it has been effective as a delivery system for epidermal growth factor and fibroblast growth factor-2 to the SCI site [14]. Therefore, this study was based on the hypothesis that collagen hydrogel acts as a delivery system for the slow release of SHED-CM to the SCI site. Thus, the study aimed to assess the effects of SHED-CM loaded in collagen hydrogel on functional recovery following SCI.

\section{Materials and Methods}

\section{Preparation of stem cell from human exfoliated de- ciduous teeth-conditioned medium}

SHEDs were obtained from the Stem Cells Technology Research Center of the University. They were cultured in Dulbecco's modified Eagle medium (DMEM; Gibco,
Grand Island, NY, USA) containing 10\% fetal bovine serum (Gibco) and antibiotics (100 U/mL penicillin, 100 $\mu \mathrm{g} / \mathrm{mL}$ streptomycin; Gibco) at $37^{\circ} \mathrm{C}$ and in $5 \% \mathrm{CO}_{2}$ atmosphere. At approximately $80 \%$ confluence, SHEDs at passages 2-4 were washed with phosphate buffer saline (Gibco) and transferred to a serum-free DMEM culture medium. After 48 hours, the CM was harvested and centrifuged at 1,500 rpm for 5 minutes. The supernatant was recentrifuged at 3,000 rpm for 3 minutes, followed by collection of the second supernatant. The CM was concentrated 10 times using ultrafiltration with a cut-off of 10 $\mathrm{kDa}$ (Millipore, Bedford, MA, USA) and stored at $-80^{\circ} \mathrm{C}$ [8-10].

\section{Preparation of collagen hydrogel}

Collagen was extracted from the rat tail based on the instructions provided in a previous study [15]. It was dissolved in $0.6 \%$ acetic acid at $4^{\circ} \mathrm{C}$ to obtain a concentration of $4 \mathrm{mg} / \mathrm{mL}$. Next, $5 \mathrm{~mL}$ of the prepared solution, $5 \mathrm{~mL}$ of DMEM or SHED-CM, and $1 \mathrm{M} \mathrm{NaOH}$ were mixed in that order to obtain collagen solutions at $\mathrm{pH} 7.5$ and a temperature of $4^{\circ} \mathrm{C}$. Final solutions containing DMEM or SHED-CM were used for injections.

\section{Animals}

Adult male Sprague-Dawley rats weighing 250-280 g were used in the present research. The animals were housed in standard conditions. The research was performed with the approval and guidelines of the Animal Care and Ethics Committee of the University (approval no., 95-11655).

\section{Experimental groups}

The rats were randomly divided into the following five groups ( $n=10 /$ group): (1) control: laminectomy was performed; (2) SCI: laminectomy and SCI were performed; (3) SCI+SHED-CM: laminectomy and SCI were performed, and SHED-CM was injected into the injury site; (4) SCI+Col: laminectomy and SCI were performed, and collagen hydrogel was injected into the injury site; and (5) SCI+SHED-CM+Col: laminectomy and SCI were performed, and SHED-CM loaded in collagen hydrogel was injected into the injury site. 


\section{Surgical procedure, injection of biomaterials, and post-surgery care}

Surgical procedures were performed under deep anesthesia with ketamine $(80 \mathrm{mg} / \mathrm{kg})$ and xylazine $(10 \mathrm{mg} / \mathrm{kg})$. In summary, laminectomy was performed at the thoracic level, and a compression injury was induced at T7 level by the application of a $20 \mathrm{~g}$ aneurysm clip (Harvard Apparatus) for 1 minute. Immediately after induction of SCI, the determinate biomaterials for each group were injected into the injury epicenter (at a depth of 1.5-2 $\mathrm{mm}$ from the dura) using a 33-gauge Hamilton syringe for a total dose of $3 \mu \mathrm{L}$. The needle was maintained in the cord for 5 minutes to prevent reflux; then, it was ejected, and the muscles and skin were sutured. After surgery, the animals were kept in a warm environment until regaining consciousness. A combination of tylosin and meloxicam was immediately injected after the surgery, followed by three additional injections for 1 day post-injury for pain relief and prevention of infection. The rats' bladders were manually expressed 3 times daily until the return of spontaneous bladder function [16].

\section{Behavioral tests}

Behavioral tests were classified as locomotor, motor, sensory, and sensory-motor to examine different aspects of behavioral performance [3]. The time of the day when the tests were performed and order of testing remained the same during the experimental period. A 30-minute interval was considered as resting time after performing different tests. Testing conditions were constant for all the tests and animals. Testing devices were cleaned after each trial.

\section{Locomotor test}

Locomotor performance was assessed using the Basso, Beattie, and Bresnahan (BBB) open-field test. Animals were acclimatized to the testing equipment and environment. All animals were examined before the surgery, on day 2 post-surgery, and then weekly up to 6 weeks. BBB testing was used to measure specific components of locomotor ability, including hind limb movement, trunk stability, toe clearance, and body, tail, and paw positions. The BBB rating scale ranged from 0 to a maximum of 21 . A score of 0 indicated no observable locomotion, and 21 indicated normal locomotor activity. All the animals were evaluated for 5 minutes by two experienced observers. The mean scores recorded from both hind paws were used for analysis [17].

\section{Motor test}

The inclined plane test was performed to assess motor function by observing muscle strength of the hind paw. All the animals were evaluated before the surgery, on day 2 post-surgery, and then weekly up to 6 weeks. The animals were placed on an inclined plane. The inclined plane was incrementally raised from the horizontal plane $\left(0^{\circ}\right)$, and the largest angle at which the animal could maintain its position for 5 seconds was recorded. Three measurements were performed for each animal, and the mean score was used for analysis [18].

\section{Sensory test}

Animals were administered the cold allodynia test. Foot withdrawal reaction to acetone contact was considered as cold sensitivity. All the animals were assessed before the surgery, on day 2 post-surgery, and then once weekly up to 6 weeks. Before starting the trial, animals could habituate inside the test apparatus (transparent cage on metal mesh) for approximately 10 minutes. A fresh acetone drop (50-100 $\mu$ ) was lightly dabbed to the mid plantar surface of both the hind paws using a syringe. It was repeated for five administrations with a minimum of 5 minutes rest between trials. Either brisk foot withdrawal or flinching was regarded as a positive reaction. The time spent for responding was measured for each animal [19].

\section{Sensory-motor test}

Animals were assessed for the sensory-motor function using the beam walk test. Assessments were performed before the surgery, at 2 weeks post-surgery, and then once weekly for up to 6 weeks. All the animals were tested on seven beams with different widths. A scoring scale, ranging from 1 to a maximum of 7 , was used according to the beam width. Rats were placed on the beams sequentially from the widest to the narrowest. Two trials were performed for each rat. The beam with the narrowest width that an animal was able to walk without foot slips was recorded [20]. 


\section{Statistical analysis}

The data are presented as the mean \pm standard deviation. Analysis of variance followed by Tukey's post-hoc test was used to compare the mean values using the IBM SPSS ver. 19.0 statistical software (IBM Corp., Armonk, NY, USA). All $p \leq 0.05$ were considered statistically significant.

\section{Results}

\section{BBB locomotor score}

All the groups had BBB scores of 21 before injury, indicating normal locomotion (Fig. 1, Table 1). The control

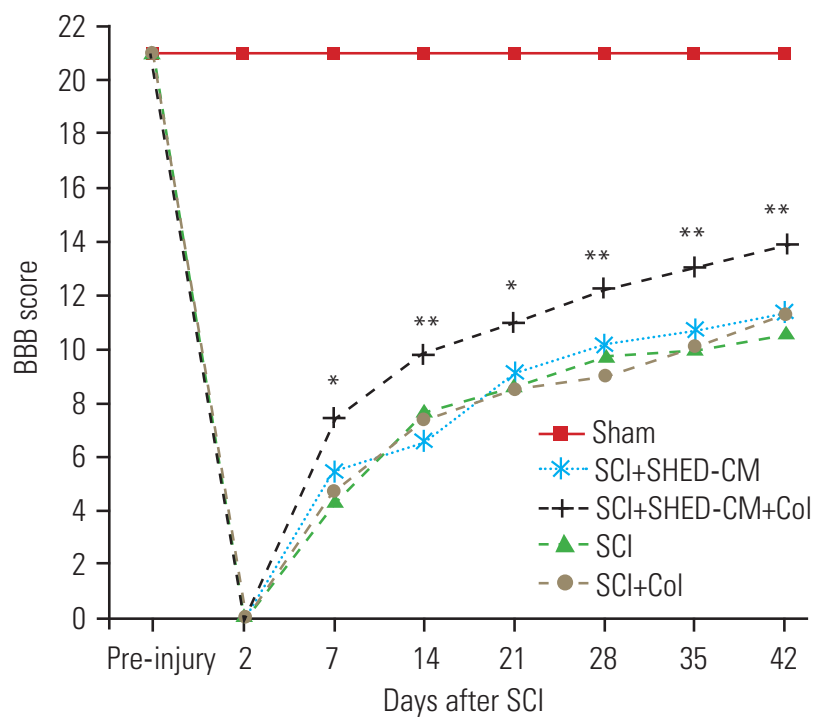

Fig. 1. BBB scores showing locomotor recovery during the study in the different groups. BBB score, Basso, Beattie, and Bresnahan score; SCI, spinal cord injury; SHED, Stem cells from human exfoliated deciduous teeth; CM, conditioned medium. ${ }^{*} p<0.05,{ }^{* *} p<0.01$ (Significant differences between the SCI+SHED-CM+Col and other injured groups are indicated). group showed a BBB score of 21 throughout the study. There were significant differences between BBB scores of the control and other groups at all time points post-surgery $(p<0.001)$. All injured animals presented flaccid hind limb paralysis with a BBB score of approximately 0 on day 2 post-surgery. Over the following 6 weeks, continuous recovery of hind limb locomotion was observed in all injured animals. However, BBB scores of the SCI+SHED$\mathrm{CM}+\mathrm{Col}$ group were significantly higher than those of the other groups beginning at 1 week post-surgery, and the difference remained significant throughout the study $(p<0.05, p<0.01)$ (Fig. 1, Table 1). Notably, no significant differences were found among SCI+SHED-CM, SCI+Col, and SCI groups. BBB score of 9 is critical to indicate a weight-supporting step. Fig. 1 shows that this stage was reached 4 days earlier in the SCI+SHED-CM+Col group compared with other injured groups (Fig. 1, Table 1).

\section{Inclined plane}

The mean angle recorded in the inclined plane test before injury was approximately $70^{\circ}$ for all the animals (Fig. 2, Table 2). The control group maintained this result throughout the study. Significant differences were observed between the control and other groups at all time points post-surgery $(p<0.001)$. After surgery, a continuous increase in the mean angle recorded in the inclined plane test was observed in all injured animals. The SCI+SHED$\mathrm{CM}+\mathrm{Col}$ group showed a significantly better performance than that by other injured groups at 2 weeks post-surgery and at all subsequent time points $(p<0.05, p<0.01)$ (Fig. 2 , Table 2). No significant differences were found among the SCI+SHED-CM, SCI+Col, and SCI groups.

Table 1. Basso, Beattie, and Bresnahan scores during the study in the different groups

\begin{tabular}{|c|c|c|c|c|c|c|c|c|}
\hline Groups & Pre-injury & Day 2 & Day 7 & Day 14 & Day 21 & Day 28 & Day 35 & Day 42 \\
\hline Sham & $21 \pm 0$ & $21 \pm 0$ & $21 \pm 0$ & $21 \pm 0$ & $21 \pm 0$ & $21 \pm 0$ & $21 \pm 0$ & $21 \pm 0$ \\
\hline $\mathrm{SCl}$ & $21 \pm 0$ & $0 \pm 0$ & $4.4 \pm 1.2$ & $7.7 \pm 0.9$ & $8.6 \pm 0.8$ & $9.8 \pm 0.9$ & $10 \pm 1.3$ & $10.6 \pm 1.6$ \\
\hline SCl+SHED-CM & $21 \pm 0$ & $0 \pm 0$ & $5.5 \pm 2.2$ & $6.6 \pm 1.7$ & $9.1 \pm 1.7$ & $10.2 \pm 1.7$ & $10.8 \pm 1.6$ & $11.4 \pm 1.9$ \\
\hline $\mathrm{SCl}+\mathrm{Col}$ & $21 \pm 0$ & $0 \pm 0$ & $4.7 \pm 0.8$ & $7.4 \pm 1.3$ & $8.5 \pm 1.6$ & $9 \pm 1.4$ & $10.1 \pm 1.2$ & $11.3 \pm 1.7$ \\
\hline SCl+SHED-CM+Col & $21 \pm 0$ & $0.2 \pm 0.4$ & $7.5 \pm 1.9^{*}$ & $9.9 \pm 1.5^{* *}$ & $11.1 \pm 1.5^{*}$ & $12.3 \pm 1.8^{* *}$ & $13.1 \pm 1.7^{* *}$ & $13.9 \pm 1.9^{* *}$ \\
\hline
\end{tabular}

Values are presented as mean \pm standard deviation.

$\mathrm{SCl}$, spinal cord injury; SHED, stem cells from human exfoliated deciduous teeth; CM, conditioned medium.

${ }^{*} p<0.05,{ }^{* *} p<0.01$ (SCI+SHED-CM+Col group vs. other injured groups). 


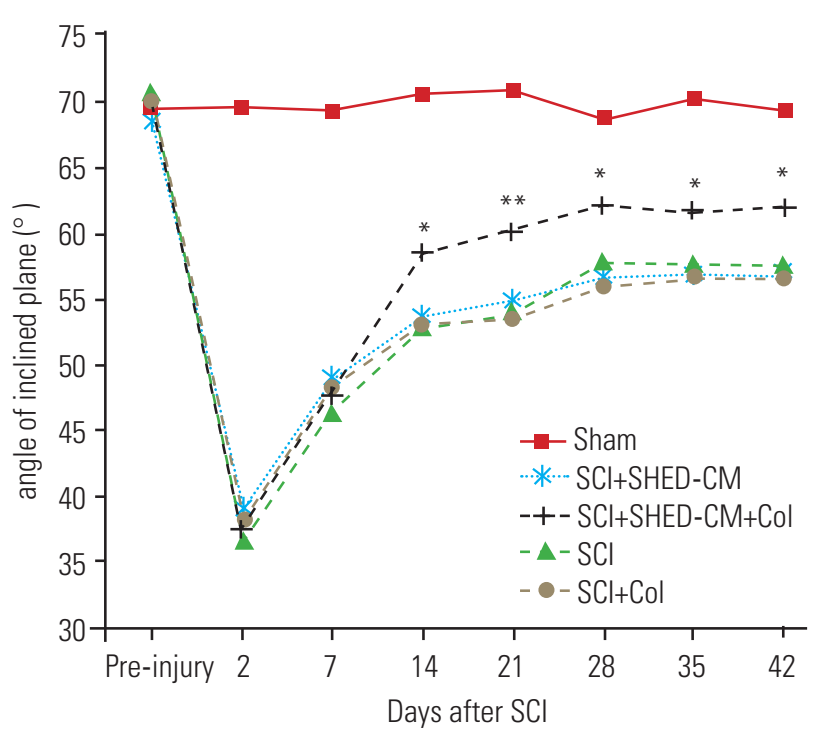

Fig. 2. The maximum angle of inclined plane at which the animal could maintain its position for 5 seconds at different time points of the study in the different groups. SCI, spinal cord injury; SHED, stem cells from human exfoliated deciduous teeth; $\mathrm{CM}$, conditioned medium. ${ }^{*} p<0.05$, ${ }^{* *} p<0.01$ (Significant differences between the SCl+SHED-CM+Col and other injured groups are indicated).

\section{Cold allodynia}

One of the distinctive features of clinical neuropathic pain is increased sensitivity to typically nonpainful cold stimuli. In our study, SCI induced adverse pain behavior of cold allodynia with positive reactions to a cold stimulus. Sensitivity to acetone contact increased at 2, 3, and 4 weeks post-injury in all the injured groups. Afterward, a decrease in sensitivity was observed at 5 and 6 weeks post-injury. The duration of response to acetone was significantly lower in the SCI+SHED-CM+Col group than in the other injured groups at 2, 3, and 4 weeks postinjury, indicating lower sensitivity $(p<0.05, p<0.01)$ (Fig. 3 , Table 3). There were no significant differences among the $\mathrm{SCI}+\mathrm{SHED}-\mathrm{CM}, \mathrm{SCI}+\mathrm{Col}$, and SCI groups. The duration of response to acetone was constant in the control group throughout the study. In addition, at the end of the study, no significant differences were observed among all the groups, indicating recovery of all animals (Fig. 3, Table 3).

Table 2. Maximum angle $\left({ }^{\circ}\right)$ of inclined plane at which the animal could maintain its position for 5 seconds at different time points of the study in the different groups

\begin{tabular}{lcrrrrrrr} 
Groups & Pre-injury & \multicolumn{1}{c}{ Day 2 } & \multicolumn{1}{c}{ Day 7 } & Day 14 & Day 21 & Day 28 & Day 35 & Day 42 \\
\hline Sham & $69.5 \pm 1.5$ & $69.6 \pm 2.2$ & $69.3 \pm 1.8$ & $70.6 \pm 1.9$ & $70.9 \pm 1.8$ & $68.7 \pm 1.8$ & $70.2 \pm 1.5$ & $69.4 \pm 0.8$ \\
SCl & $70.7 \pm 1.8$ & $36.4 \pm 3.9$ & $46.4 \pm 3.9$ & $52.8 \pm 3.6$ & $53.8 \pm 2.6$ & $57.8 \pm 3.6$ & $57.7 \pm 3.3$ & $57.6 \pm 3.2$ \\
SCl+SHED-CM & $68.6 \pm 1.7$ & $39 \pm 3.8$ & $49 \pm 3.8$ & $53.7 \pm 2.9$ & $54.9 \pm 2.8$ & $56.6 \pm 1.7$ & $57.1 \pm 2.9$ & $56.9 \pm 2.9$ \\
SCl+Col & $70.2 \pm 1.5$ & $38.2 \pm 4.6$ & $48.2 \pm 4.6$ & $53 \pm 2.8$ & $53.4 \pm 2.5$ & $56 \pm 2.9$ & $56.6 \pm 3.4$ & $56.4 \pm 1.8$ \\
\hline SCl+SHED-CM+Col & $70.1 \pm 2.1$ & $37.6 \pm 5.2$ & $47.6 \pm 5.2$ & $58.4 \pm 2.5^{*}$ & $60 \pm 2.9^{* *}$ & $62.2 \pm 2.3^{*}$ & $61.7 \pm 2.5^{*}$ & $62 \pm 4.1^{*}$ \\
\hline
\end{tabular}

Values are presented as mean \pm standard deviation.

$\mathrm{SCl}$, spinal cord injury; SHED, stem cells from human exfoliated deciduous teeth; CM, conditioned medium.

${ }^{*} p<0.05,{ }^{* *} p<0.01$ (SCI+SHED-CM+Col group vs. other injured groups).

Table 3. Duration of response (sec) to cold stimulus (acetone) at different time points of the study in the different groups

\begin{tabular}{lcccccccc} 
Groups & Pre-injury & Day 2 & Day 7 & Day 14 & Day 21 & Day 28 & Day 35 & Day 42 \\
\hline Sham & $4.3 \pm 1.5$ & $4.1 \pm 1.3$ & $4.2 \pm 0.9$ & $4.2 \pm 1.5$ & $4.3 \pm 1.9$ & $4.2 \pm 1.3$ & $3.9 \pm 1.1$ & $4 \pm 1.8$ \\
SCl & $3.5 \pm 1.1$ & $1.8 \pm 1.3$ & $1.8 \pm 1.4$ & $9.2 \pm 2.8$ & $8.8 \pm 2.3$ & $9 \pm 2.4$ & $6.6 \pm 2.1$ & $5.8 \pm 2.2$ \\
\hline SCI+SHED-CM & $3.8 \pm 1.6$ & $1.8 \pm 1.8$ & $2.1 \pm 1.1$ & $8.9 \pm 2.7$ & $8.9 \pm 2.5$ & $8.5 \pm 2.1$ & $6.2 \pm 1.3$ & $6 \pm 1.8$ \\
\hline SCl+Col & $4.3 \pm 1.6$ & $1.7 \pm 1.3$ & $1.5 \pm 1.5$ & $9.4 \pm 3.2$ & $9.5 \pm 2.3$ & $9.7 \pm 2.9$ & $6.7 \pm 3.1$ & $5.5 \pm 2.2$ \\
\hline SCl+SHED-CM+Col & $4 \pm 1.1$ & $1.8 \pm 1.4$ & $1.7 \pm 1.2$ & $4.4 \pm 1.8^{* *}$ & $5.2 \pm 2.2^{* *}$ & $5.3 \pm 2.1^{*}$ & $5.8 \pm 1.5$ & $5.3 \pm 1.7$ \\
\hline
\end{tabular}

Values are presented as mean \pm standard deviation.

SCI, spinal cord injury; SHED, stem cells from human exfoliated deciduous teeth; CM, conditioned medium.

${ }^{*} p<0.05,{ }^{* *} p<0.01$ (SCl+SHED-CM+Col group vs. other injured groups). 


\section{Beam walk}

Before injury, all rats were able to cross the beam with the narrowest width without any errors in foot placement (Fig. 4, Table 4). The control group showed a maximum beam walk score of 7 throughout the study. All injured groups showed severe deficits following injury. The beam walk score was significantly higher in the SCI+SHED-CM+Col group than in the other injured groups beginning at 4 weeks post-injury and during the subsequent weeks $(p<0.05, p<0.01)$ (Fig. 4, Table 4). No significant differences were observed among the SCI+SHED-CM, SCI+Col, and SCI groups.

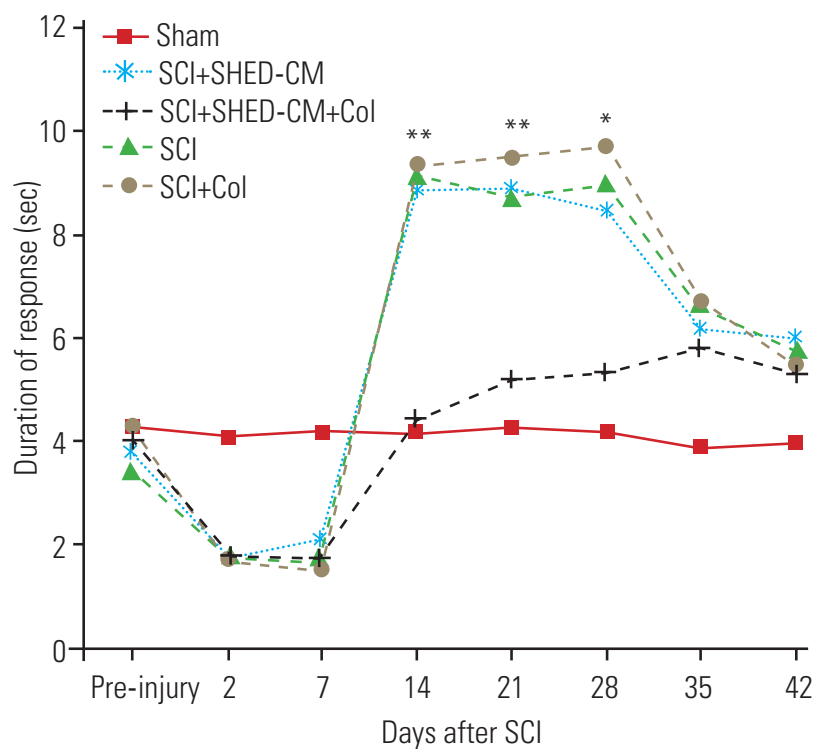

Fig. 3. Duration of response to cold stimulus (acetone) showing cold allodynia at different time points of the study in the different groups. $\mathrm{SCl}$, spinal cord injury; SHED, stem cells from human exfoliated deciduous teeth; CM, conditioned medium. ${ }^{*} p<0.05,{ }^{* *} p<0.01$ (Significant differences between the SCl+SHED-CM+Col and other injured groups are indicated).

\section{Discussion}

The purpose of this study was to evaluate the possible therapeutic effects of SHED-CM loaded in collagen hydrogel on performance improvement when injected at the site of SCI in rats.

Previous investigations have dealt with the transplantation of SHEDs and their beneficial effects on functional recovery after experimental SCI [6,7]. For example, grafted SHEDs resulted in improved motor activity following SCI [7]. However, few research studies have specifically focused on the therapeutic potential of SHED-CM to treat SCI.

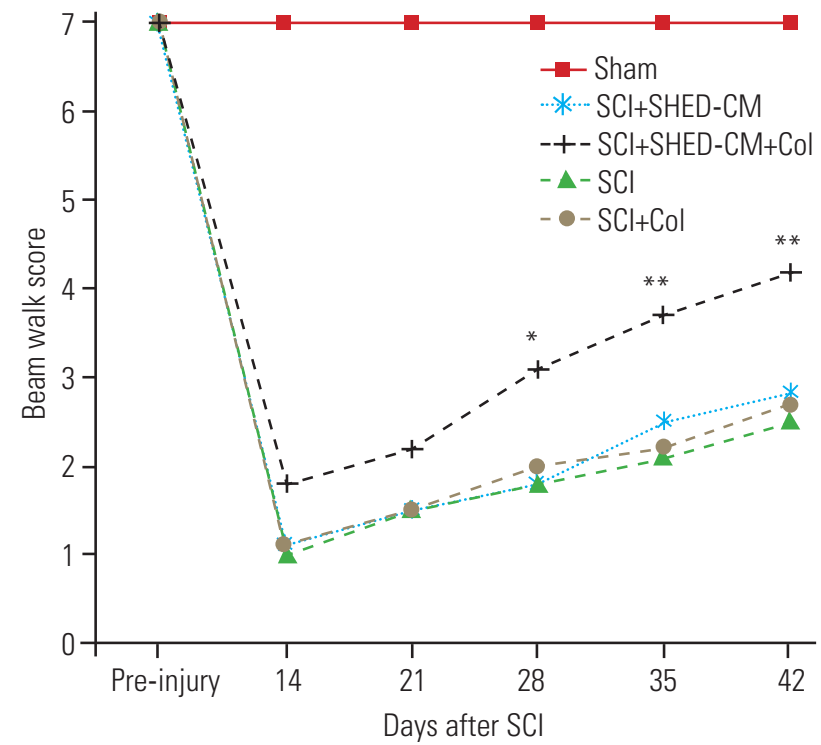

Fig. 4. Beam walk scores during the study in the different groups. SCl, spinal cord injury; SHED, stem cells from human exfoliated deciduous teeth; CM, conditioned medium. ${ }^{*} p<0.05,{ }^{* *} p<0.01$ (Significant differences between the $\mathrm{SCl}+\mathrm{SHED}-\mathrm{CM}+\mathrm{Col}$ and other injured groups are indicated).

Table 4. Beam walk scores during the study in the different groups

\begin{tabular}{lcccccc} 
Groups & Pre-injury & Day 14 & Day 21 & Day 28 & Day 35 & Day 42 \\
Sham & $7 \pm 0$ & $7 \pm 0$ & $7 \pm 0$ & $7 \pm 0$ & $7 \pm 0$ & $7 \pm 0$ \\
SCl & $7 \pm 0$ & $1 \pm 0.9$ & $1.5 \pm 0.7$ & $1.8 \pm 1$ & $2.1 \pm 0.8$ & $2.5 \pm 0.8$ \\
SCl+SHED-CM & $7 \pm 0$ & $1.1 \pm 0.7$ & $1.5 \pm 0.7$ & $1.8 \pm 0.7$ & $2.5 \pm 0.5$ & $2.8 \pm 0.7$ \\
SCl+Col & $7 \pm 0$ & $1.1 \pm 0.7$ & $1.5 \pm 0.8$ & $2 \pm 0.8$ & $2.2 \pm 0.6$ & $2.7 \pm 0.6$ \\
SCl+SHED-CM+Col & $7 \pm 0$ & $1.8 \pm 1.1$ & $2.2 \pm 0.6$ & $3.1 \pm 0.5^{*}$ & $3.7 \pm 0.9^{* *}$ & $4.2 \pm 0.7^{* *}$ \\
\hline
\end{tabular}

Values are presented as mean \pm standard deviation.

SCI, spinal cord injury; SHED, stem cells from human exfoliated deciduous teeth; CM, conditioned medium.

${ }^{*} p<0.05,{ }^{* *} p<0.01$ (SCl+SHED-CM+Col group vs. other injured groups) 
Our results demonstrated for the first time that intraspinal injection of SHED-CM loaded in collagen hydrogel can promote functional recovery following SCI. These results suggested a potential area for investigating the mechanisms in future in vitro and in vivo studies. The findings are consistent with previous studies that reported that trophic factors released by dental pulp stem cells improve proliferation, migration, differentiation, and survival of neurons [21,22]. In addition, recent studies have reported that CM derived from dental pulp stem cells has beneficial effects on bone healing [9], cognitive function in Alzheimer's disease [10], and cardiac injury [8].

SHED-CM contains various factors that have antiinflammatory and anti-apoptotic activity as well as neuroprotective and angiogenic effects. For example, brainderived neurotrophic factor improves the survival of neurons and plasticity [23] and positively affects learning and memory formation [24]. Glial cell line-derived neurotrophic factor is involved in neuroprotection [25], suppression of microglial activation [23], and improvement in axonal regeneration $[25,26]$. Stem cell factor and hepatocyte growth factor are also involved in neuroprotection $[27,28]$.

SHED-released factors are used as a concentrated CM to optimize potential impacts. We consider that the effects of SHED-CM on functional recovery after SCI may be attributed to simultaneous effects of the factors in SHEDCM. In the present study, intraspinal injection of collagen hydrogel alone could not improve performance after SCI. This result is consistent with the findings in earlier studies showing little or no effect following collagen hydrogel implantation at the SCI site [29]. Conversely, collagen hydrogel loaded with other factors has been found to be beneficial for the recovery after SCI, similar to the results found in this study [11-13]. Therefore, our results confirmed that collagen hydrogel acts as an excellent delivery system for the slow release of SHED-CM to the injured spinal cord.

According to our data, SHED-CM alone did not affect performance improvement. This result may be due to the rapid diffusion of SHED-CM after injection into the spinal cord. However, SHED-CM was effective when loaded into collagen hydrogel. This result is explainable by the fact that collagen hydrogel acts as a slow-release system. Our results confirmed the findings of previous reports regarding the effectiveness of collagen scaffold as a drug delivery system in SCI [11-13].

It is also possible that SHED-CM without collagen is ef- fective if higher doses and/or more injections are administered. A previous study showed that the use of CM from bone marrow-derived mesenchymal stem cells for 1 week with a higher dose than that used in our study was effective on motor function after SCI [30]. One advantage of our study compared with this earlier study is that different aspects of behavior, including locomotor, motor, sensory, and sensory-motor functions, were investigated. Second, using a single injection of a lower dose of SHED-CM loaded in collagen was effective, and this could be more applicable for future clinical studies.

There are some limitations of our study. Only behavioral tests were performed, and no histological analysis was included. There was no dose oscillation and analysis of inflammation and neuronal protection. Finally, considering the importance of functional recovery in terms of a therapeutic approach for SCI, it was important to find a method for using behavioral tests after SCI for subsequent research, such as histological study and in vitro analysis of SHED-CM. Therefore, further investigations are currently being implemented to clarify the mechanisms involved in this study.

\section{Conclusions}

Our data demonstrated that intraspinal administration of SHED-CM loaded in collagen hydrogel is advantageous following compressive SCI in rats and leads to improved functional recovery. Therefore, our data proposes a cellfree therapeutic approach for SCI.

\section{Conflict of Interest}

No potential conflict of interest relevant to this article was reported.

\section{Acknowledgments}

This article was extracted from $\mathrm{PhD}$ thesis written by Reza Asadi-Golshan, and financially supported from Shiraz University of Medical Sciences, Shiraz, Iran (grant no. 9511655). The research was performed at Cellular and Molecular Medicine Student Research Group, Experimental and Comparative Medical Center, Anatomy Department, Shiraz University of Medical Sciences, Shiraz, Iran. 


\section{ORCID}

Reza Asadi-Golshan: https://orcid.org/0000-0003-1901-7171

Vahid Razban: https://orcid.org/0000-0002-8966-6081

Esmaeil Mirzaei: https://orcid.org/0000-0002-0176-5123

Abdolkarim Rahmanian: https://orcid.org/0000-0001-5223-7083

Sahar Khajeh: https://orcid.org/0000-0003-1614-2962

Zohreh Mostafavi-Pour: https://orcid.org/0000-0002-3779-177X

Farzaneh Dehghani: https://orcid.org/0000-0003-2217-5672

\section{Author Contributions}

Conception and design: RAG, EM, VR, FD; data acquisition: RAG, AR, SK; data analysis and interpretation of data: RAG, FD; drafting of the manuscript: RAG, AR; critical revisions: ZMP, VR, EM, FD; and final approval: all authors.

\section{References}

1. Hagg T, Oudega M. Degenerative and spontaneous regenerative processes after spinal cord injury. J Neurotrauma 2006;23:264-80.

2. Muir GD, Webb AA. Mini-review: assessment of behavioural recovery following spinal cord injury in rats. Eur J Neurosci 2000;12:3079-86.

3. Sedy J, Urdzikova L, Jendelova P, Sykova E. Methods for behavioral testing of spinal cord injured rats. Neurosci Biobehav Rev 2008;32:550-80.

4. Kerkis I, Kerkis A, Dozortsev D, et al. Isolation and characterization of a population of immature dental pulp stem cells expressing OCT-4 and other embryonic stem cell markers. Cells Tissues Organs 2006;184:105-16.

5. D’Aquino R, De Rosa A, Laino G, et al. Human dental pulp stem cells: from biology to clinical applications. J Exp Zool B Mol Dev Evol 2009;312B:408-15.

6. Sakai K, Yamamoto A, Matsubara K, et al. Human dental pulp-derived stem cells promote locomotor recovery after complete transection of the rat spinal cord by multiple neuro-regenerative mechanisms. J Clin Invest 2012;122:80-90.

7. Nicola FC, Rodrigues LP, Crestani T, et al. Human dental pulp stem cells transplantation combined with treadmill training in rats after traumatic spinal cord injury. Braz J Med Biol Res 2016;49:e5319.

8. Yamaguchi S, Shibata R, Yamamoto N, et al. Dental pulp-derived stem cell conditioned medium reduces cardiac injury following ischemia-reperfusion. Sci Rep 2015;5:16295.

9. Fujio M, Xing Z, Sharabi N, et al. Conditioned media from hypoxic-cultured human dental pulp cells promotes bone healing during distraction osteogenesis. J Tissue Eng Regen Med 2017;11:2116-26.

10. Mita T, Furukawa-Hibi Y, Takeuchi H, et al. Conditioned medium from the stem cells of human dental pulp improves cognitive function in a mouse model of Alzheimer's disease. Behav Brain Res 2015;293:189-97.

11. Han Q, Sun W, Lin H, et al. Linear ordered collagen scaffolds loaded with collagen-binding brain-derived neurotrophic factor improve the recovery of spinal cord injury in rats. Tissue Eng Part A 2009;15:292735.

12. Han Q, Jin W, Xiao Z, et al. The promotion of neural regeneration in an extreme rat spinal cord injury model using a collagen scaffold containing a collagen binding neuroprotective protein and an EGFR neutralizing antibody. Biomaterials 2010;31:9212-20.

13. Liang W, Han Q, Jin W, et al. The promotion of neurological recovery in the rat spinal cord crushed injury model by collagen-binding BDNF. Biomaterials 2010;31:8634-41.

14. Jimenez Hamann MC, Tator CH, Shoichet MS. Injectable intrathecal delivery system for localized administration of EGF and FGF-2 to the injured rat spinal cord. Exp Neurol 2005;194:106-19.

15. Rajan N, Habermehl J, Cote MF, Doillon CJ, Mantovani D. Preparation of ready-to-use, storable and reconstituted type I collagen from rat tail tendon for tissue engineering applications. Nat Protoc 2006;1:2753-8.

16. Rivlin AS, Tator CH. Effect of duration of acute spinal cord compression in a new acute cord injury model in the rat. Surg Neurol 1978;10:38-43.

17. Basso DM, Beattie MS, Bresnahan JC. A sensitive and reliable locomotor rating scale for open field testing in rats. J Neurotrauma 1995;12:1-21.

18. Rivlin AS, Tator CH. Objective clinical assessment of motor function after experimental spinal cord injury in the rat. J Neurosurg 1977;47:577-81.

19. Choi Y, Yoon YW, Na HS, Kim SH, Chung JM. Behavioral signs of ongoing pain and cold allodynia in a rat model of neuropathic pain. Pain 1994;59:369-76. 
20. Von Euler M, Akesson E, Samuelsson EB, Seiger A, Sundstrom E. Motor performance score: a new algorithm for accurate behavioral testing of spinal cord injury in rats. Exp Neurol 1996;137:242-54.

21. Nosrat IV, Widenfalk J, Olson L, Nosrat CA. Dental pulp cells produce neurotrophic factors, interact with trigeminal neurons in vitro, and rescue motoneurons after spinal cord injury. Dev Biol 2001;238:120-32.

22. Arthur A, Shi S, Zannettino AC, Fujii N, Gronthos S, Koblar SA. Implanted adult human dental pulp stem cells induce endogenous axon guidance. Stem Cells 2009;27:2229-37.

23. Lu B, Gottschalk W. Modulation of hippocampal synaptic transmission and plasticity by neurotrophins. Prog Brain Res 2000;128:231-41.

24. Ozawa T, Yamada K, Ichitani Y. Hippocampal BDNF treatment facilitates consolidation of spatial memory in spontaneous place recognition in rats. Behav Brain Res 2014;263:210-6.

25. Konishi Y, Yang LB, He P, et al. Deficiency of GDNF receptor GFRal in Alzheimer's neurons results in neuronal death. J Neurosci 2014;34:13127-38.
26. Iannotti C, Li H, Yan P, Lu X, Wirthlin L, Xu XM. Glial cell line-derived neurotrophic factor-enriched bridging transplants promote propriospinal axonal regeneration and enhance myelination after spinal cord injury. Exp Neurol 2003;183:379-93.

27. Niimura M, Takagi N, Takagi K, et al. The protective effect of hepatocyte growth factor against cell death in the hippocampus after transient forebrain ischemia is related to the improvement of apurinic/ apyrimidinic endonuclease/redox factor-1 level and inhibition of NADPH oxidase activity. Neurosci Lett 2006;407:136-40.

28. Li JW, Li LL, Chang LL, Wang ZY, Xu Y. Stem cell factor protects against neuronal apoptosis by activating AKT/ERK in diabetic mice. Braz J Med Biol Res 2009;42:1044-9.

29. Nomura H, Tator CH, Shoichet MS. Bioengineered strategies for spinal cord repair. J Neurotrauma 2006;23:496-507.

30. Cantinieaux D, Quertainmont R, Blacher S, et al. Conditioned medium from bone marrow-derived mesenchymal stem cells improves recovery after spinal cord injury in rats: an original strategy to avoid cell transplantation. PLoS One 2013;8:e69515. 